\title{
Cardiovascular tissue banking activity during SARS-CoV-2 pandemic: evolution of national protocols and Lombardy experience
}

\author{
Giorgio Mastroiacovo (D) Anna Guarino - Sergio Pirola • Marco Gennari • \\ Francesca Capriuoli • Barbara Micheli • Alice Bonomi • Giuseppe Piccolo • \\ Luca Dainese - Gianluca Polvani
}

Received: 16 July 2021 / Accepted: 3 September 2021 / Published online: 14 September 2021

(C) The Author(s), under exclusive licence to Springer Nature B.V. 2021

\begin{abstract}
The worldwide pandemic outbreak due to severe acute respiratory syndrome-coronavirus-2 (SARS-CoV-2) has created unprecedented challenges for public health services. Lombardy, region of the Northern Italy, has been the first area in the Western world whose organs and tissues procurement programs have had to face the virus pandemic emergency. We retrospectively collected and analyzed data about cardiovascular tissues (CT) in 2019 and in 2020 . We aimed to describe the rapid evolution of SARS-CoV-2 regulation laws for tissue donor's selection and
\end{abstract}

G. Mastroiacovo $(\bowtie) \cdot$ S. Pirola $\cdot$ M. Gennari ·

L. Dainese · G. Polvani

Department of Cardiovascular Surgery, IRCCS Centro

Cardiologico Monzino, Via Parea 4, 20138 Milan, Italy

e-mail: gio.mastroiacovo@hotmail.it;

Sergio.pirola@ccfm.it

A. Guarino - F. Capriuoli · B. Micheli

Cardiovascular Tissue Bank of Lombardy, Centro

Cardiologico Monzino IRCCS, Milan, Italy

\footnotetext{
A. Bonomi

Department of Statistics, Centro Cardiologico Monzino, IRCCS, Milan, Italy

G. Piccolo

Regional Transplant Coordination, Lombardy Region,

Milan, Italy

G. Polvani

Department of Biomedical Surgical and Dental Sciences,

University of Milan, Milan, Italy
}

harvesting from February 2020 until January 2021. As expected the number of CT donors in 2020 was significantly lower than those of 2019 (66 vs. 99, $p$ value 0.02 ). The total number of CT collected from donors have been 254 in 2019 and 206 in 2020 ( $p$ 0.28). Femoral arteries were the most required vascular tissues (55.5\% in 2019 and $40 \%$ in 2020). Fifty-five and forty-eight pulmonary valves were implanted in 2019 and 2020, respectively. No differences were found for the types of $\mathrm{CT}$ requests between the 2 years. The median age of receivers of vascular tissues was $69.6 \pm 14.6$ years in the 2019 and $63.3 \pm 14.9$ years in $2020(p<0.01)$. The median age of receivers of pulmonary and aortic valves did not differ between the 2 years $(9.32 \pm 11.49$ vs. $8.36 \pm 10.66$ and $48.67 \pm 27.19$ vs. $37.14 \pm 31.97$ respectively). Despite the dramatically reduction of donors, the number of CT collected has not decreased significantly and so far the CT distribution rate is comparable to those of 2019.

Keywords Cardiovascular tissue harvesting . SARS-CoV-2 pandemic · Cardiovascular tissues donation
Abbreviations
CNT
Centro nazionale trapianti
CT
Cardiovascular tissues
GMP
Good manufacture practice
NPS 
SARS-CoV-2 Syndrome-coronavirus-2

\section{Introduction}

The worldwide pandemic due to severe acute respiratory syndrome-coronavirus-2 (SARS-CoV-2) has created unprecedented challenges for public health services.

Italy, in particular its northern area, Lombardy, was the first European country to be hit (Istituto Superiore di Sanità Sorveglianza Integrata COVID-19 in Italia, aggiornamento del 04 Gennaio 2021 Giugno 2020. Available at :https://www.epicentro.iss.it/coronavirus/sars-cov-2-dashboard).

At the date of 19 February 2021, there have been 2.780 .882 cases with 95.235 fatalities due to SARSCoV-2 all over Italy; of these, 574.000 (27.971 fatalities) have occurred in the Lombardy region (Available at: Coronavirus in Lombardia, tutti gli aggiornamenti in diretta (lombardianotizie.online).

Lombardy was the first region whose tissues procurement programs have had to face the virus pandemic emergency (Istituto Superiore di Sanità Sorveglianza Integrata COVID-19 in Italia, aggiornamento del 04 Gennaio 2021 Giugno 2020. Available at :https://www.epicentro.iss.it/coronavirus/sars-cov-2dashboard).

Data on heterologous cardiovascular grafts' management in the SARS-CoV-2 era are very limited.

Italian Transplant Authority (Centro Nazionale Trapianti, CNT) released guidelines on cardiovascular tissues (CT) management requiring real-time reverse transcription polymerase chain reaction (rRT-PCR) assays on nasopharyngeal swab (NPS) or broncho alveolar lavage $24-48 \mathrm{~h}$ before organs retrieval, to identify and exclude possible SARS-CoV-2 positive donors (Available at: Regione Lombardia GiuntaDirezione Generale Welfare Prevenzionewww.regionelombardia.it).

It is obvious that such a dramatic scenario poses many new questions and challenges. Aim of this paper is to report our experience as Cardiovascular Tissue Bank of the Lombardy region of Italy during the 2020, reporting Italian guidelines and management that have been changed according to the evolving situation of the pandemic outbreak and the commitment of cardiovascular tissues.

\section{The Lombardy cardiovascular tissue bank}

The Centro Cardiologico Monzino hospital is the Regional headquarters of the cardiovascular tissue bank since 1992; its aim is to procure, process, evaluate, decontaminate, cryopreserve and distribute CT obtained from heart/non- heart beating donors according to CNT and European guidelines.

The tissue bank staffs, composed by cardiac surgeons, biologists, perfusionists and laboratory technicians, is involved h24/365 days in CT's procurement in all Lombardy region, tissue processing in class A/B GMP (good manufacture practice), validation, cryopreservation, storage and distribution in Italy and Europe (Conferenza permanente per I rapporti tra lo stato, le regioni e le province autonome di Trento e Bolzano Rep. Atti n. 66/CSR seduta dell'8 Marzo 2018).

Indications for cardiac valvular tissue use have been released for both the pediatric and the adult cardiac surgery patients. The pulmonary valve is mainly used in pediatric cardiac operation (i.e. congenital cyanogen cardiomyopathy) (Stark 1998) while aortic valves are generally used in the adult population for infective processes (i.e. endocarditis) or structural valve deterioration of previously implanted bioprosthesis (Musci et al. 2010). Vascular tissues are usually required to replace infected vascular prosthesis or to perform vascular bypasses in case of acute ischemia and malignant infiltrating tumors (Furlough et al. 2019).

\section{Material and methods}

We have collected data from our bank and compared the activity of Lombardy region between 2019 and 2020; specifically, we compared the number of cardiovascular tissues collected and distributed. The protocol and procedures employed for this study were ethically reviewed and approved by Centro Cardiologico Monzino's ethical committee. 
Statistical analysis

Categorical variables were presented as the mean frequencies and percentage. Differences between 2019 versus 2020 were evaluated by paired Chisquare Test of Fisher, as appropriate. The trends in the months were measured by ANOVA.

Tests were two-sided. $P$ values $<0.05$ were considered statistically significant. Analyses were performed by SAS version 9.4 (SAS Institute Inc., Cary, NC).

\section{Results}

CNT donors'tissues recruitment guidelines

\section{Phase I}

In the first pandemic phase (from January to February 2020) the CNT reccomended to collect a careful patient's history of travel in areas considered endemic for SARS-CoV2 by WHO in the 28 days prior to donation. In such cases, diagnostic tests for SARS$\mathrm{CoV}-2$ were performed on donors; in case of positivity, the donor would have been excluded from the donation program.

Starting from February 24th, Lombardy has been considered endemic area. From February 27th the number of endemic regions increased until the whole of Italy was considered an endemic area in the beginning of March.

\section{Phase II}

Since the beginning of March 2020, the recommendation indicated by CNT was to perform a specific test for SARS-CoV-2 (an oral or nasopharyngeal swab) collected within $24 \mathrm{~h}$ from death on all eligible donors reported by Lombardy hospital's ICU. The swab had to be sent to the laboratory as soon as possible and no later than $24 \mathrm{~h}$ since its collection.

The results on respiratory secretions had to be available before tissues' collection. In case of a positive result, the tissues had to be considered unsuitable for donation.
Phase III

From mid-March 2020, due to the higher sensitivity compared to the oro- or NPS, it was recommended the execution of the specific test for the search of SARSCoV-2 on respiratory secretions collected from bronchiolo-alveolar lavage or from deep bronchial aspirate. When possible, the sample had to be collected in 24 , maximum $48 \mathrm{~h}$ prior to tissue harvesting. In case of a positive result, the tissues had to be considered unsuitable for donation.

\section{Phase IV}

Since May 26th, the CNT established that donors with documented positive history of SARS-CoV-2 or a positive history of undocumented SARS-CoV-2 (symptoms compatible with SARS-CoV-2 without certain and lab-certified exclusion of contagion) would have an unacceptable risk level and have been therefore excluded from the program.

On the other hand, a positive history of close contact with patients affected by SARS-CoV-2 in absence of clinical symptoms and with a negative NPS performed within 24 to $48 \mathrm{~h}$ prior donation, required the tissues to be collected at least 4 weeks after the last risk contact. In this case the risk level of infected tissues would have considered standard.

\section{Phase V}

From the end of June onwards, the CNT decided to divide the donors in three classes:

- Class 1 positive history of documented SARSCoV-2.

Indication donor tissues may be harvested at least 4 weeks after documented virologic recovery (resolution of symptoms and 2 consecutives negative NPS at least $24 \mathrm{~h}$ apart) and with a negative NPS for SARS-CoV-2 performed within 24 to $48 \mathrm{~h}$ prior to harvest.

- Class 2 a positive history of undocumented COVID (symptomatology compatible with SARS-CoV-2 but no or negative swabs or serologically positive antibodies to SARS-CoV-2) 
Indications donor tissues may be harvested at least 4 weeks after documented virological cure (see above).

- Class 3 positive history of close contact with SARS-CoV-2 patients in absence of clinical symptoms or with a negative NPS.

Indication donor tissues may be retrieved at least 4 weeks after the last risk contact and a negative NPS performed within 24 to $48 \mathrm{~h}$ prior to retrieval.

Lombardy tissue bank's series of donors and receivers

The number of donors in 2020 (66) was significantly lower than the one recorded in 2019 (99) ( $p$ value 0,02). Figure 1 shows the trend of donors between 2019 and 2020; it followed the lockdown phases of the pandemic, reaching the lowest value in the months from February to April and November to December. The total number of CT collected from donors have been 254 in 2019 and 206 in 2020 ( $p$ 0.28) (Fig. 2). Figure 3 shows the numbers and trend of CT recipients in the same period. The trend curves of CT requests and donors overlap, with a minimum of requests in the months from February to April and in November and December. It should be remarked that the peak of requests was in January, when SARS-CoV-2 infection in Italy was not yet mentioned, and in October, the month before the second national lockdown, that coincided with the resume of the full hospital surgical activity. The total amount of CT receivers was 107 in 2019 and 112 in 2020 ( $p$ 0.73).

In 2019 femoral arteries grafts were the most required vascular tissues $(25 / 45,55.5 \%)$, followed by abdominal aorta among with the two iliac arteries (8/ $45,17.8 \%)$, thoracic aorta $(7 / 45,15.5 \%)$, vein cava $(2 /$ $45,4.4 \%)$, saphenous vein $(2 / 45,4.4 \%)$ and femoral vein $(1 / 45,2.2 \%)$, (Table 1). In 2020 the femoral arteries accounted for the $40 \%$ of vascular tissues' requests (22/55), followed by thoracic aorta $14 / 55$ (25.4\%), the abdominal aorta among with the two iliac arteries $7 / 55(12.7 \%)$, vein cava $5 / 55(9 \%)$, saphenous vein $4 / 55(7.3 \%)$ and femoral vein $3 / 55(5.5 \%)$ (Table 1). Seven aortic valves $(11 \%)$ and fifty-five pulmonary valves $(89 \%)$ were used in 2019 , while in 2020 were used nine aortic valves (16\%) and fortyeight pulmonary valves $(84 \%)$ respectively (Table 1 ).

Tables 2 and 3 depict the reasons for which vascular and valvular tissues have been allocated respectively in 2019 and 2020. No differences were found for the types of requests, but the demand for vascular tissues for substitutions of segments infiltrated by tumors increased in 2020 compared to 2019, even if it is not statistically significant $(p 0.11)$.

The median age of receivers of vascular homograft was $69.6 \pm 14.6$ years in the 2019 and $63.3 \pm 14.9$ years in $2020(p<0.01)$. The median age of receiver of pulmonary valves was $9.32 \pm 11.49$ years and $8.36 \pm 10.66$ in the 2019 and 2020, respectively ( $p$ value 0.59 ), while the median age of receiver of aortic valves was

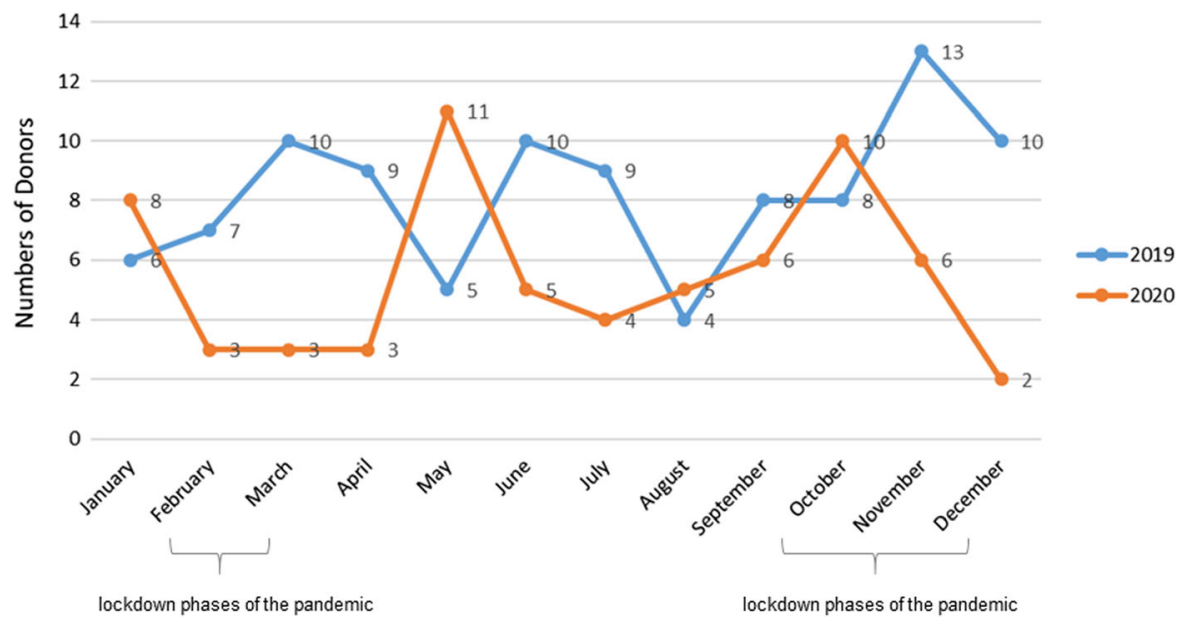

Fig. 1 Comparison of cardiovascular tissue donors in Lombardy between 2019 and 2020 


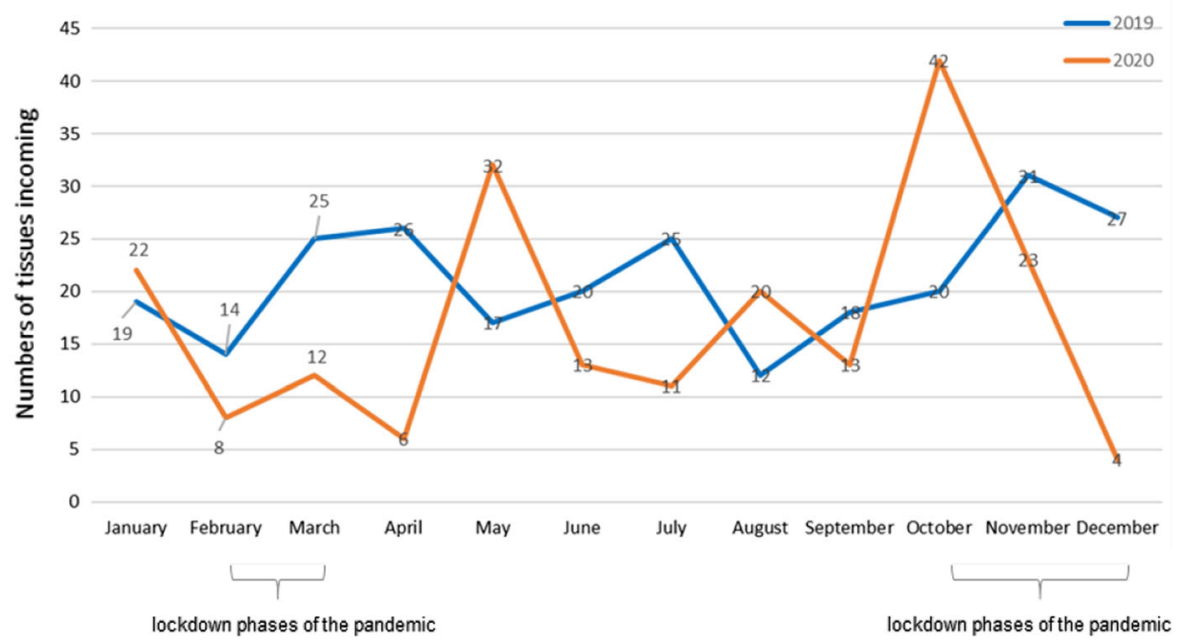

Fig. 2 Comparison of cardiovascular tissues harvested between 2019 and 2020

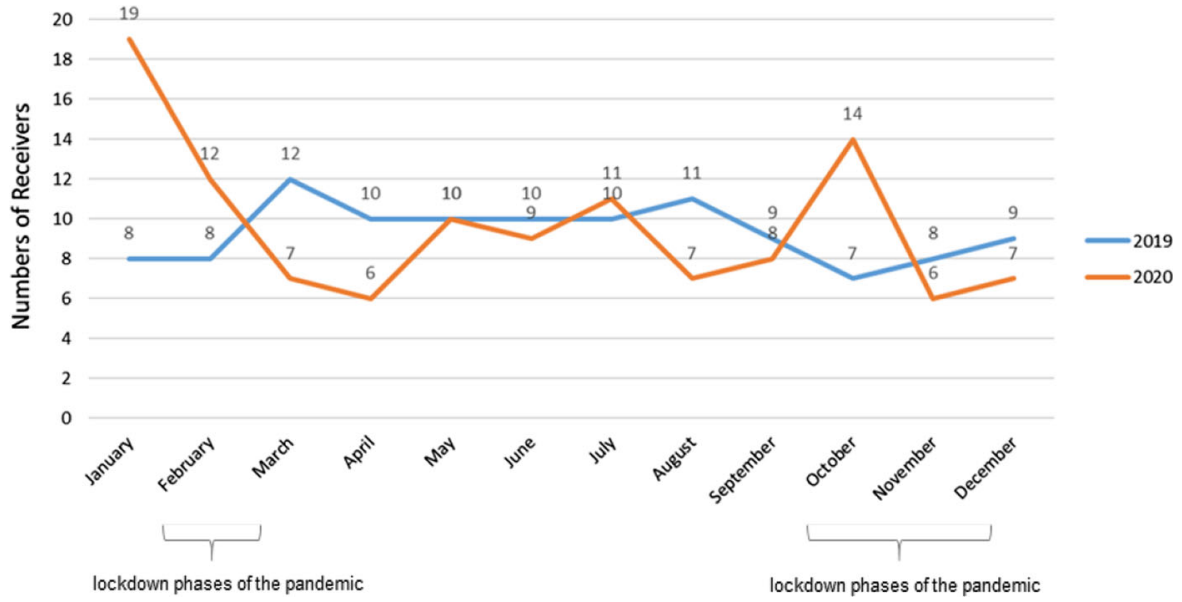

Fig. 3 Comparison of cardiovascular tissue requests in Italy between 2019 and 2020

Table 1 Cardiovascular tissues requested to our tissue bank in 2019 and 2020

\begin{tabular}{lccl}
\hline Variable & 2019 (tot.107) & 2020 (tot.112) & $p$ value $(<0.05)$ \\
\hline Pulmonary valve & $55(51.4 \%)$ & $48(42.8 \%)$ & 0.62 \\
Femoral artery & $25(23.3 \%)$ & $22(19.6 \%)$ & 0.50 \\
Abdominal aorta among with iliac arteries & $8(7.5 \%)$ & $7(6.2 \%)$ & 0.72 \\
Thoracic aorta & $7(6.5 \%)$ & $14(12.5 \%)$ & 0.13 \\
Aortic valve & $7(6.5 \%)$ & $9(8 \%)$ & 0.52 \\
Cava vein & $2(1.9 \%)$ & $5(4.5 \%)$ & 0.27 \\
Saphenous vein & $2(1.9 \%)$ & $4(3.5 \%)$ & 0.44 \\
Femoral vein & $1(0.9 \%)$ & $3(2.7 \%)$ & 0.33 \\
\hline
\end{tabular}

$48.67 \pm 27.19$ years in the 2019 and $37.14 \pm 31.97$ in the 2020, respectively ( $p 0.41)$.
In 2019 our tissue bank received 58 requests for vascular tissues and 69 in 2020. 
Table 2 Causes of vascular tissue implantation in 2019 and 2020

\begin{tabular}{lccc}
\hline Variable & 2019 (tot.45) & 2020 (tot.55) & $p$ value $(<0.05)$ \\
\hline Reinterventions of infected vascular prostheses & $17(37.8 \%)$ & $21(38.1 \%)$ & 0.96 \\
Vascular bypass for obliterating arteriopathy or acute ischemia & $11(24.4 \%)$ & $12(22 \%)$ & 0.76 \\
Substitutions of vascular segments infiltrated by tumours & $7(15.6 \%)$ & $16(29 \%)$ & 0.11 \\
Corrections of fistulas & $5(11.1 \%)$ & $2(3.6 \%)$ & 0.24 \\
Pseudoaneurysms correction & $2(4.4 \%)$ & $2(3.6 \%)$ & 1.0 \\
Mycotic aortic aneurysm & $2(4.4 \%)$ & $2(3.6 \%)$ & 1.0 \\
Vascular laceration with haemorrhage & $1(2.2 \%)$ & $0(0 \%)$ & 0.45 \\
\hline
\end{tabular}

Table 3 Causes of heart valves implantation in 2019 and 2020

\begin{tabular}{llll}
\hline Variable & 2019 (tot.62) & 2020 (tot.57) & $p$ value $(<0.05)$ \\
\hline Aortic valve disease & $17(27.4 \%)$ & $20(35 \%)$ & 0.37 \\
Pulmonary valve disease & $16(26 \%)$ & $13(23 \%)$ & 0.70 \\
Aorta Vessel Interventions & $8(13 \%)$ & $4(7 \%)$ & 0.37 \\
Endocarditis & $8(13 \%)$ & $5(8.7 \%)$ & 0.47 \\
Hypoplastic Left heart syndrome & $6(9.7 \%)$ & $9(16 \%)$ & 0.32 \\
Double-Oulet Right Ventricle & $2(3.2 \%)$ & $1(1.8 \%)$ & 1.0 \\
Atrioventricular Channel & $2(3.2 \%)$ & $0(0 \%)$ & 0.50 \\
Tetralogy of Fallot & $2(3.2 \%)$ & $3(5.2 \%)$ & 0.67 \\
Great Vessels Transposition & $2(3.2 \%)$ & $0(0 \%)$ & 0.50 \\
Troncus arteriosus & $0(0 \%)$ & $2(3.5 \%)$ & 0.23 \\
\hline
\end{tabular}

We managed to supply the $76 \%(45 / 58)$ and $80 \%$ $(55 / 69)$ of the requests in 2019 and 2020, respectively. Concerning the heart valves, we were able to supply the $50 \%(62 / 125)$ and the $56 \%(57 / 102)$ of the requests in 2019 and 2020, respectively.

\section{Discussion}

Despite the full commitment of the healthcare providers, since the first appearance of the SARSCoV-2 outbreak there has been an overall decrease of about $10 \%$ in the number of annual donors in Italy (with this value in some Nations exceeding 20\%) and $8 \%$ in the number of transplantations (Available at www.trapianti.salute.gov.it).

As reported above, the tissue bank was able to cover the same number of CT requirements between 2019 and 2020, with a decrease in heart valves requests in 2020 due to a reduction in regular hospital activity because of the SARS-CoV-2 pandemic.
From February to June 2020, the regulation on cardiovascular tissues' retrieval from donors was amended five times to face the ongoing pandemic.

What described above underlines how critical has been the lack of knowledge about the virus and its behavior for the spreading of the infection and, at the same time, the continuous efforts aimed to revise and correct the guidelines as long as new scientific data became available.

In the period March-April 2020, CT procurement underwent a significant reduction, due to hospital personal reorganization and the decrease in both the number of patients eligible for donation and deaths for road and work-related accidents for national lockdown.

Figure 1 shows that from 99 donors in 2019, the trend was down to 66 in 2020 with a statistically significant difference between the two years.

The decrease of donors during the months of lockdown was not accompanied by the same decrease in tissue procurement, since CT harvesting is more 
related to the donors 'type and age than to their amount.

Indeed, in the case of a multi-tissue provider, more CT are collected from a single donor compared to a multi-organ supplier. Moreover, the younger the donor, the more $\mathrm{CT}$ are available for harvesting.

As shown in Fig. 1 the trend of donors has followed the lockdown phases of the pandemic, reaching the lowest value in the months from February to April and November to December. The trend of CT distribution and donors overlap, with a minimum of requests in the months from February to April and in November and December (Fig. 2). It should be remarked that the peak of demands was in January, when SARS-CoV-2 infection in Italy was not yet mentioned, and in October, the month before the second national lockdown, that coincided with the resume of the full hospital surgical activity.

The decline in the number of donors and in the request for $\mathrm{CT}$ during the months of national lockdown risks, once the hospitals and healthcare facilities have restored their own full operative capacity, to create a critical unbalance between demand and offer of CT in dramatically favor of the first one.

Indeed, tissue demands have been higher in October 2020 than in the previous year, due to the number of patients who probably received surgical indication during the pandemic phase who could not be treated at the most critical moment.

However, our analyses did not show any statistically significant differences in the numbers of receivers between 2019 and 2020.

Two main conditions consented this result.

The first point consists in the extraordinary effort sustained by the Italian medical healthcare system to increase as much as possible its ordinary working activity to compensate the reduction in surgical schedule during the national lockdown period.

The second reason is related to the supplies of CT collected over the years and cryopreserved in the Tissue Bank.

The pandemic status and the national lockdown have created delays in both diagnosis and surgical treatment of cancer (Vecchio et al. 2020), leading patients to undergo surgery at more advanced stages of the oncological pathologies (Garg et al. 2020).

This would explain why there has been an increase in the use of vascular tissues to replace tumorinfiltrated arterial/venous vessels in 2020. The mean age of patients who undergo CT implantation for substitutions of vascular segments infiltrated by tumours is lower than those who receive CT for other causes. This would clarify why in 2020 the median age for vascular homograft implantation was significantly lower compared to 2019, according to our analyses.

However, we do not yet know how long this pandemic will persist, and it is consequently necessary to find alternative solutions to deal with the increased demand of CT when the pandemic ends.

One possible solution is to increase donor recruitment by reconsidering the CNT's donor guidelines. Indeed, to date, there is no evidence reported in the literature regarding infection with SARS-CoV-2 in CT recipients and many questions remain unanswered.

Does SARS-CoV-2 remain active and potentially infectious even in CT treated with antibiotics and subsequently cryopreserved?

Currently, we do not know if a tissue harvested from a SARS-CoV-2 positive donor even once treated and cryopreserved is able to transmit the virus once transplanted (Paggiaro 2021).

Unfortunately, there are just few studies about the effects of cryopreservation on SARS-CoV-2.

In a recent published paper by $\mathrm{R}$ Jashari et al. it is suggested that clinical application of the valves from two SARS-CoV-2 donors presents a negligible risk for novel coronavirus transmission and that the release of a pulmonary valve (negative with the SARS-CoV-RTPCR) harvested from a heart with dilated obstructive cardiomyopathy would have been considered favorably for a life-threatening situation when no other similar allografts were available (Jashari et al. 2021).

As the number of $\mathrm{CT}$ donors continues to decline due to the need to exclude SARS-CoV-2 -positive donors, studies need to be conducted as soon as possible to answer this question.

In addition, operations requiring the use of $\mathrm{CT}$ are often not post-ponable.

The literature shows that about $81 \%$ of people with SARS-CoV-2 have mild disease (Weiss and Murdoch 2020; Wu and McGoogan 2020) and never require hospitalization and that age, diabetes mellitus, male sex, chronic obstructive pulmonary disease and hypercholesterolemia are independent risk factors associated with mortality (Li et al. 2020). Grasselli et al. demonstrated that hospital and ICU mortality rates were respectively 12 and 27 per 1000 patients-days on a cohort of 3988 consecutive critically ill patients with 
laboratory-confirmed COVID-19 referred for ICU admission (Grasselli et al. 2020).

So, the question is: in a life-threating scenario, would it be more useful, for the patient, to receive a CT retrieved from a SARS-CoV-2-positive donor, even if there is a risk of infection, or not? Would the mortality rate for a patient undergoing operations requiring CT be higher if he/she did not undergo the operation or if he/she received a CT from a SARS-CoV-2-positive donor?

In addition, we do not know whether implanting a CT from a SARS-CoV-2-positive donor into a recipient who has never been infected or that has no SARSCoV-2 antibodies at the time of operation, even if previously infected by the virus, will result in an active infection.

Moreover, since CT recipients are not subjected to immunomodulatory therapy, we do not know, if the virus were to be active, whether it would trigger a major infection as important as those observed in some patients undergone cardiac transplantation (Caraffa et al. 2020).

Controlled-randomized clinical trial and large multicentric studies are required to answer these questions.

Since studies on the persistence of SARS-CoV-2 infectivity in cryo-preserved tissues require time, one possibility would be, given the need for CT and the progressive reduction of stocks, to start harvesting tissues from SARS-Co V-2 positive patients.

It could then be decided to implant CT collected from SARS-CoV-2 positive donors in positive patients or vaccinated or immunized against SARS-CoV-2, especially in those cases whose surgery is lifesaving.

Moreover, it is necessary to set up a reorganization of health structures and care pathways considering the need to ensure continuity of care for the many patients (often of pediatric age) waiting for a cardiac or vascular tissue implant.

Study limitation

This is a retrospective study with all the limitations connected to it.

\section{Conclusion}

The fast spreading of SARS-CoV-2 infection poses new questions and debate for the healthcare systems worldwide.

Italy was the second country after China that had to face SARS-CoV-2 pandemic when not much was known about this virus yet. As we know, this is the first report about cardiovascular tissues harvesting activity and numbers in SARS-Co V-2 era available in the literature.

We described the guidelines that Italian national health authority is being implemented for cardiovascular tissues procurement.

Despite the reduction of donors'tissues, so far the distribution rate of CT is the same as in the previous years and we are confident for the future since in these first two months of 2021 we collected 52 CT. However, since we do not know what will happen in the near future, there may be alternative ways to regulate $\mathrm{CT}$ distribution such as demonstrating that the virus does not survive after decontamination and cryopreservation or starting to harvest CT from SARS$\mathrm{CoV}-2+$ to treat SARS-CoV-2 + or vaccinated patients.

In conclusion, considering the latest data from the literature, CT procurement from asymptomatic SARSCoV-2 positive patients, died for non- SARS-CoV-2related causes, should be considered, providing the investigation for SARS-CoV-2 RNA with RT-PCR in the tissue.

All RNA-negative tissues may be considered valid for distribution.

Funding none.

\section{Declarations}

Conflicts of interest The author that they have declare no conflict of interest.

Data availability This manuscript has associated data in a data repository, including all data for which data deposition is mandatory.

\section{References}

Available at www.trapianti.salute.gov.it

Available at: Coronavirus in Lombardia, tutti gli aggiornamenti in diretta (lombardianotizie.online) 
Available at: Regione Lombardia Giunta- Direzione Generale Welfare Prevenzione- www.regionelombardia.it

Caraffa R, Bagozzi L, Fiocco A et al (2020) Coronavirus disease 2019 (COVID-19) in the heart transplant population: a single-centre experience. Eur $\mathrm{J}$ Cardiothorac Surg 58(5):899-906

Conferenza permanente per I rapporti tra lo stato, le regioni e le province autonome di Trento e Bolzano Rep. Atti n. 66/CSR seduta dell'8 Marzo 2018

Del Vecchio Blanco G, Calabrese E, Biancone L et al (2020) The impact of COVID-19 pandemic in the colorectal cancer prevention. Int J Colorectal Dis 35(10):1951-1954

Furlough CL, Jain AK, Ho KJ et al (2019) Peripheral artery reconstructions using cryopreserved arterial allografts in infected fields. J Vasc Surg 70(2):562-568

Garg PK, Kaul P, Choudhary D et al (2020) Cancer surgery in the era of COVID-19 pandemic: changing dynamics. J Surg Oncol. https://doi.org/10.1002/jso.26156

Grasselli G, Greco M, Zanella A et al (2020) COVID-19 lombardy ICU network risk factors associated with mortality among patients with COVID-19 in intensive care units in lombardy. Italy AMA Intern Med 180(10):1345-1355

Jashari R, Van Esbroeck M, Vanhaebost J et al (2021) The risk of transmission of the novel coronavirus (SARS-CoV-2) with human heart valve transplantation: evaluation of cardio-vascular tissues from two consecutive heart donors with asymptomatic COVID-19. Cell Tissue Bank 9:1-10
Li X, Xu S, Yu M et al (2020) Risk factors for severity and mortality in adult COVID-19 inpatients in Wuhan. J Allergy Clin Immunol 146(1):110-118

Musci M, Weng Y, Hübler M et al (2010) Homograft aortic root replacement in native or prosthetic active infective endocarditis: twenty-year single-center experience. $\mathrm{J}$ Thorac Cardiovasc Surg 139(3):665-673

Paggiaro AO, Carvalho VF, Gemperli R (2021) Effect of different human tissue processing techniques on SARS-CoV2 inactivation-review. Cell Tissue Bank 22(1):1-10

Stark J (1998) The use of valved conduits in pediatric cardiac surgery. Pediatr Cardiol 19(4):282-288

Istituto Superiore di Sanità Sorveglianza Integrata COVID-19 in Italia, aggiornamento del 04 Gennaio 2021 Giugno 2020. Available at: https://www.epicentro.iss.it/coronavirus/ sars-cov-2-dashboard

Weiss P, Murdoch DR (2020) Clinical course and mortality risk of severe COVID-19. Lancet 395(10229):1014-1015

Wu Z, McGoogan JM (2020) Characteristics of and important lessons from the coronavirus disease 2019 (COVID-19) outbreak in China: summary of a report of 72314 cases from the Chinese center for disease control and prevention. JAMA 323:1239

Publisher's Note Springer Nature remains neutral with regard to jurisdictional claims in published maps and institutional affiliations. 